\title{
Analysis of layered composite beam to underwater shock including structural damping and stiffness effects
}

\author{
S.W. Gong and K.Y. Lam \\ Institute of High Performance Computing, 1 Science Park Road, \#01-01, The Capricorn Singapore Science Park II, \\ Singapore 117528
}

Received 9 January 2001

Revised 14 November 2002

\begin{abstract}
This paper deals with the transient response of layered composite beams subjected to underwater shock. The Doubly Asymptotic Approximation (DAA) method is employed in this study to treat the fluid-structure interactions. The effective structural damping and stiffness are formulated and incorporated in the fluid-structure-coupled equations, which relate the structure response to fluid impulsive loading and are solved using coupled finite-element and DAA-boundary element codes. The present computational method facilitates the study of transient response of the layered composite beams to underwater shock, involving the effects of structural damping and stiffness. In addition, the effect of free surface on the transient response of the layered beam to underwater shock is examined.
\end{abstract}

\section{Introduction}

The transient response of a composite structure to an underwater shock is a complex problem due to the interaction between the fluid and structure. A variety of surface interaction approximations have been developed and utilized for this problem. The most promising approximation proposed appears to be the Double Asymptotic Approximation [1] (DAA). McLoy and Sun [2] presented analysis of a thick-section composite cylinder subjected to underwater blast loading. In their study, the DAA was used and implemented in the finite element code ABAQUS through a user subroutine to solve their problem. Gong and Lam [3] presented a study on the transient response of stiffened composite submersible hull subjected to underwater shock loading using coupled finite element and DAAboundary element code. Lam et al. [4,5] presented their study on transient response of two-layered elastic and orthotropic cylindrical shells exposed to underwater shock. In their study, the Reflected-AfterflowVirtual-Source (RAVS) was used to model the fluid- structure interaction. After that, Gong and Lam [6] also presented their work on the transient response of a floating composite ship section subjected to underwater shock.

As listed above, efforts have been made to analyze composite plates and shells subjected to underwater shock. However, all these studies did not consider the effects of structural damping on the transient response and thus an analysis procedure that includes the structural damping has not been dealt with yet. Since structural damping can be used to attenuate transient response of the target layered structures to underwater shock, a comprehensive study of structural damping effects is necessary for optimal design layered structures. The present work addresses this and presents a computational procedure to study the influence of effective damping and stiffness of the layered structure on its transient response to underwater shock. The DAA method is also employed in this study to describe the fluid-structure interactions, which is included the effects of effective structural damping and stiffness and also involved the effects of the free surface. Informa- 
tion on the effects of effective damping and stiffness on the transient response is useful in designing layered structures to enhance their resistance to underwater shock.

\section{Effective structural damping and stiffness}

In a ship design, an effective way to attenuate transient response of the ship to underwater shock is to use a layer of damping material coating on the ship hull. Two typical forms of bottom hulls, $V$ and flat bottom hulls, are often used. To simplify the study, a twolayered beam is modeled as shown in Fig. 1 to study the effects of damping and stiffness. The beam, simplysupported at its two ends, was submerged in seawater and subjected to explosive shock loading generated by a charge of TNT. The charge is detonated underwater with a distance of $R$ from the beam. The two layer composite beam consists of a base elastic layer and a damping layer. The former corresponds to the bottom hull while the latter corresponds to the coating layer. The base elastic layer has a thickness of $h_{1}$, Young's modulus $E_{1}$ and loss factor $\eta_{1}$ and the damping layer has a thickness of $h_{2}$, Young's modulus $E_{2}$ and loss factor $\eta_{2}$. The beam has a common length of $L$ and width of $W$ for both base elastic and damping layers.

A rectangular Cartesian coordinate system is used as shown in Fig. 1. The fluid density and sound speed are $1025 \mathrm{~kg} / \mathrm{m}^{3}$ and $1500 \mathrm{~m} / \mathrm{s}$ respectively. The effective structural loss factor $\eta_{s t}$ is derived using the complex stiffness method that was proposed by Oberst [7]; while the effective Young's modulus $E_{s t}$ is derived using the minimum potential energy principle.

\subsection{Effective structural loss factor for the two layer composite beam}

In this study, the energy dissipating effects of the material damping are considered and thus the Young's moduli for the base layer and damping layer are complex numbers that are denoted by a superscript star. The neutral axis position can be estimated by

$$
\delta=\frac{1}{2} \frac{E_{1}^{*} h_{1}^{2}-E_{2}^{*} h_{2}^{2}}{E_{1}^{*} h_{1}+E_{2}^{*} h_{2}}
$$

In view of the fact that the loss factor in the base elastic layer is much lower than that in the damping layer, i.e., $\eta_{1} \ll \eta_{2}$, the effect of $\eta_{1}$ can be ignored and thus the above equation becomes

$$
\delta=\frac{1}{2} \frac{h_{1}-h_{2} \lambda_{e}^{*} \lambda_{h}}{\left(1+\lambda_{e}^{*} \lambda_{h}\right)}
$$

where

$$
\lambda_{e}^{*}=\frac{E_{2}^{*}}{E_{1}}=\frac{E_{2}}{E_{1}}\left(1+j \eta_{2}\right)=\lambda_{e}\left(1+j \eta_{2}\right)
$$

and $\lambda_{h}=\frac{h_{2}}{h_{1}}, \lambda_{e}=\frac{E_{2}}{E_{1}}$.

Since the loss factor of the base elastic layer is very small, we assume that $E_{1} \approx E_{1}^{*}$. The complex bending stiffness can then be calculated by

$$
\begin{aligned}
B^{*} & =\int_{-\left(h_{1}-\delta\right)}^{\delta} E_{1} y^{2} d y+\int_{\delta}^{h_{2}+\delta} E_{2}^{*} y^{2} d y \\
& =B_{1} \frac{1+2 \lambda_{e}^{*}\left(2 \lambda_{h}+3 \lambda_{h}^{2}+2 \lambda_{n}^{3}\right)+\lambda_{e}^{* 2} \lambda_{h}^{*}}{\left(1+\lambda_{e}^{*} \lambda_{h}\right)}
\end{aligned}
$$

Where $B_{1}=E_{1} I_{1}$, and $I_{1}$ is the moment of inertia of the base elastic layer. The effective loss factor and the complex bending stiffness for the layered beam is related by

$$
B^{*}=B_{s t}\left(1+j \eta_{s t}\right)
$$

Substituting Eq. (5), in conjunction with Eq. (3), into Eq. (4) yields

$$
\begin{aligned}
& \frac{B_{s t}\left(1+j \eta_{s t}\right)}{B_{1}} \\
= & {\left[1+2 \lambda_{e}\left(1+j \eta_{2}\right)\left(2 \lambda_{h}+3 \lambda_{h}^{2}+2 \lambda_{h}^{3}\right)\right.} \\
& \left.+\lambda_{e}^{2}\left(1+j \eta_{2}\right)^{2} \lambda_{h}^{4}\right] /\left[1+\lambda_{e}+\lambda_{h}\left(1+j \eta_{2}\right)\right]
\end{aligned}
$$

Taking the imaginary component of both side of Eq. (6) to be equal yields

$$
\begin{gathered}
\frac{\eta_{s t}}{\eta_{2}}=\frac{\lambda_{e} \lambda_{h}}{\left(1+\lambda_{e} \lambda_{h}\right)} \\
\times \frac{3+6 \lambda_{h}+4 \lambda_{h}^{2}+2 \lambda_{e} \lambda_{h}^{3}+\lambda_{e}^{2} \lambda_{h}^{4}}{1+2 \lambda_{e}\left(2 \lambda_{h}+3 \lambda_{h}^{2}+2 \lambda_{h}^{3}\right)+\lambda_{e}^{2} \lambda_{h}^{4}}
\end{gathered}
$$

Equation (7) shows that the effective structural loss factor $\eta_{s t}$ can be obtained from the loss factor of the damping layer if the Young's modulus ratio and the thickness ratio of the damping and the base layers are known.

\subsection{Effective Young's modulus for the two layer composite beam}

Consider the differential element of the two-layer beam subjected to constant unidirectional strain $\varepsilon_{x}=\varepsilon$, for which the potential energy per unit is

$$
U=\frac{1}{2} E_{s t} \varepsilon^{2}
$$


Underwater Shock Wave

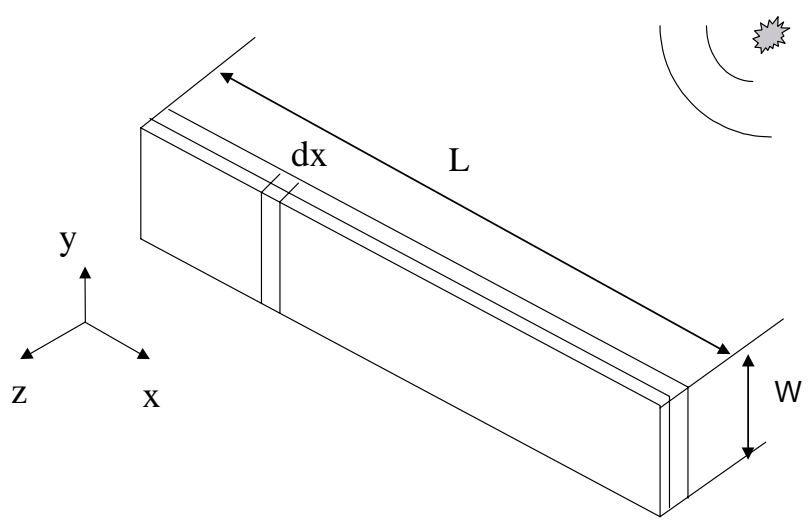

(a)

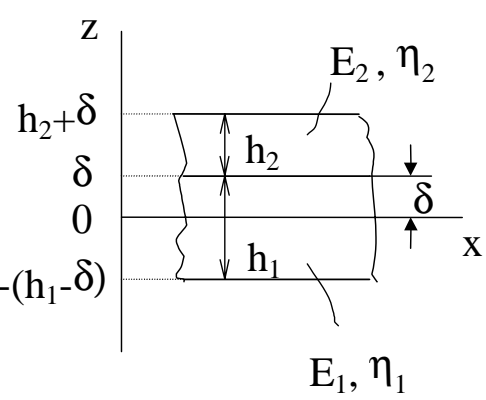

(b)

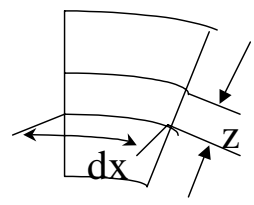

(c)

Fig. 1. Description of the problem, layered beam geometry and coordinator system.

and assume

$$
\varepsilon_{y}=\varepsilon_{z}=\nu_{s t} \varepsilon
$$

Where $E_{s t}$ and $\nu_{s t}$ are the effective Young's modulus and the Poisson ratio of the layered composite beam respectively. $\varepsilon_{x}, \varepsilon_{y}$ and $\varepsilon_{z}$ are the stains respectively in $x$-, $y$ - and $z$-directions. The relation of strain and stress is expressed by

$$
\begin{aligned}
& \sigma_{x}=\left[\lambda\left(1+2 \nu_{s t}\right)+2 G\right] \varepsilon \\
& \sigma_{y}=\sigma_{z}=\left[\lambda\left(1+2 \nu_{s t}\right)+2 G \nu_{s t}\right] \varepsilon
\end{aligned}
$$

where

$$
\lambda=\frac{E_{s t} \nu_{s t}}{\left(1+\nu_{s t}\right)\left(1-2 \nu_{s t}\right)}, G=\frac{E_{s t}}{2\left(1+\nu_{s t}\right)} .
$$

Let $\nu_{1}$ and $\nu_{2}$ denote the Poisson ratios of the damping layer and the base elastic layer respectively and $\sigma_{i x}, \sigma_{i y}, \sigma_{i z}$ denote the stresses respectively in the $x$-, $y$-, $z$-directions and in the two layers $(i=1,2)$. For the possible displacement state, the strain energy density is

$$
\begin{aligned}
U^{(s)}= & \frac{1}{2}\left(\sigma_{1 x} \varepsilon_{x}+\sigma_{1 y} \varepsilon_{y}+\sigma_{1 z} \varepsilon_{z}\right) \frac{h_{1}}{h} \\
& +\frac{1}{2}\left(\sigma_{2 x} \varepsilon_{x}+\sigma_{2 y} \varepsilon_{y}+\sigma_{2 z} \varepsilon_{z}\right) \frac{h_{2}}{h} \\
= & \frac{1}{2} \varepsilon^{2}\left[\frac{1-\nu_{1}+4 \nu_{1} \nu_{s t}+2 \nu_{s t}^{2}}{1-\nu_{1}-2 \nu_{1}^{2}} E_{1} \frac{h_{1}}{h}\right. \\
& \left.+\frac{1-\nu_{2}+4 \nu_{2} \nu_{s t}+2 \nu_{2}^{2}}{1-\nu_{2}-2 \nu_{2}^{2}} E_{2} \frac{h_{2}}{h}\right]
\end{aligned}
$$

For a minimum $U^{(s)}$, the coefficient $\nu_{s t}$ can be selected from $\frac{\partial U^{(s)}}{\partial \nu_{s t}}=0$ and $\frac{\partial^{2} U^{(s)}}{\partial \nu_{s t}^{2}}>0$ as follows

$$
\begin{aligned}
& \nu_{s t}= \\
& \frac{\left(1-\nu_{1}-2 \nu_{1}^{2}\right) \nu_{2} \lambda_{e} \lambda_{h}+\left(1-\nu_{2}-2 \nu_{2}^{2}\right) \nu_{1}}{\left(1-\nu_{1}-2 \nu_{1}^{2}\right) \lambda_{e} \lambda_{h}+\left(1-\nu_{2}-2 \nu_{2}^{2}\right)}
\end{aligned}
$$

Based on the minimum potential energy principle, $U^{(s)} \geqslant U$, from Eqs (8) and (12) the effective Young's modulus can be obtained

$$
E_{s t}=\left(\frac{1-\nu_{1}+4 \nu_{1} \nu_{s t}+2 \nu_{s t}^{2}}{1-\nu_{1}-2 \nu_{1}^{2}}\right.
$$




\section{Loss factor ratio, $\eta_{s i} / \eta_{2}$}

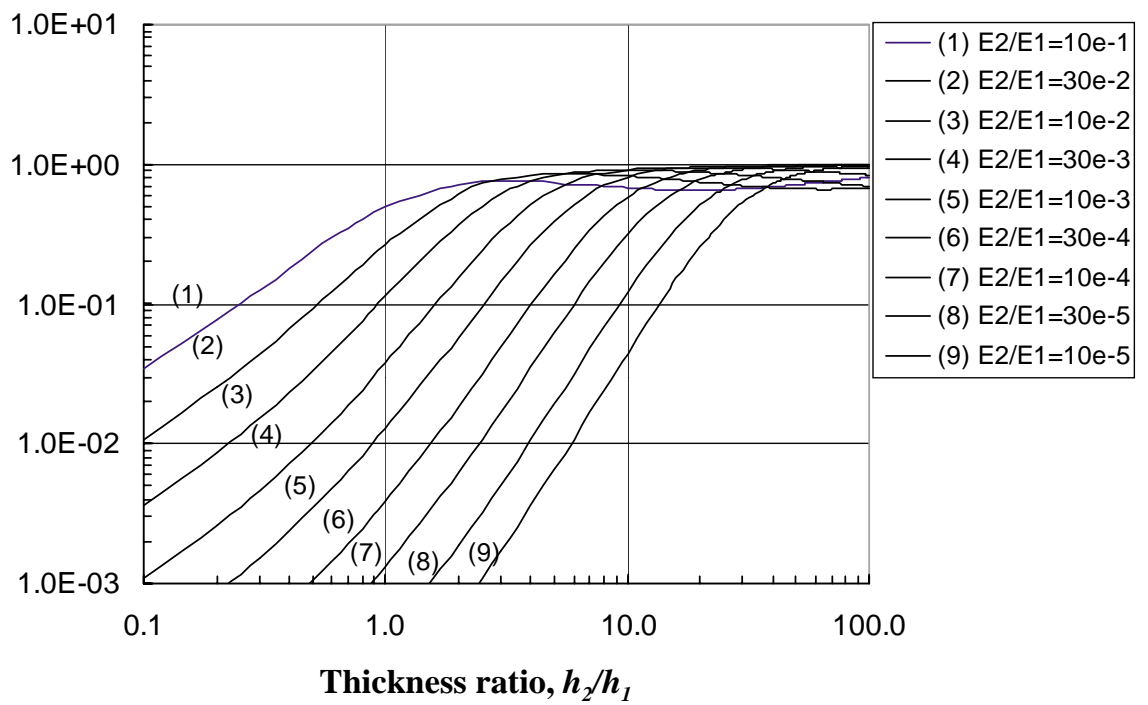

Fig. 2. Variations of loss factor ratio with thickness ratio.

$$
\left.+\frac{1-\nu_{2}+4 \nu_{2} \nu_{s t}+2 \nu_{2}^{2}}{1-\nu_{2}-2 \nu_{2}^{2}} \lambda_{e} \lambda_{h}\right) E_{1} \frac{h_{1}}{h}
$$

For the case of $\nu_{1}=\nu_{2}=\nu_{s t}$, Eq. (14) can be simplified as

$$
E_{s t}=\left(1+\lambda_{e} \lambda_{h}\right) E_{1} \frac{h_{1}}{h}
$$

\section{Computational procedure of layered beam to underwater shock}

Based on Doubly Asymptotic Approximation (DAA) method, the coupled fluid-structure interaction for the two-layered beam can be expressed as $[1,8]$

$$
\begin{aligned}
& {\left[M_{s t}\right]\{\ddot{x}\}+\left\lfloor C_{s t}^{*}\right\rfloor\{\dot{x}\}+\left\lfloor K_{s t}^{*}\right\rfloor\{x\} } \\
= & -\left[G_{1}\right]\left[A_{f}\right]\left(\left\{p_{1}\right\}+\left\{p_{s}\right\}\right) \\
& {\left[M_{f}\right]\left\{\dot{p}_{S}\right\}+\rho c\left[A_{f}\right]\left\{p_{S}\right\} } \\
= & \rho c\left[M_{f}\right]\left(\left[G_{t}\right]^{T}\{\ddot{x}\}-\left\{\dot{u}_{1}\right\}\right)
\end{aligned}
$$

where $\left[M_{s t}\right],\left[C_{s t}^{*}\right]$ and $\left[K_{s t}^{*}\right]$ are the structural mass, damping and stiffness matrices. The structural damping and stiffness coefficients $C_{s t}^{*}$ and $K_{s t}^{*}$ in the coupled fluid-structure interaction equations for the twolayered beam are determined by the effective structural loss factor $\eta_{s t}$ and the effective Young's modulus $E_{s t}$. $\{x\}$ is the vector of unknown nodal displacements and rotations and $\{\ddot{x}\}$ is the nodal acceleration vector. $\left\{p_{1}\right\}$ is the incident nodal pressure vector and $\left\{p_{S}\right\}$ is the scattered-wave pressure vector. $\left[A_{f}\right]$ is the diagonal area matrix associated with elements in the fluid mesh and $\left[G_{t}\right]$ is the transformation matrix that relates the structural and fluid nodal surface forces. $\left[M_{f}\right]$ is the symmetric fluid mass matrix for the fluid-surface mesh. $\rho$ and $c$ are the density and sound velocity of the fluid.

To solve the coupled fluid-structure interaction equations, coupled finite element and DAA boundary element codes were employed. Structural calculations were carried out using the finite element code while the fluid-structure interaction was handled by the DAA boundary element code. The finite element code used in this study was LS-DYNA3D $[9,10]$. The DAA boundary element method code used was USA [8] (Underwater Shock Analysis). Prior to the calculation of USA/LS-DYNA3D, the structural damping and stiffness are calculated using a Fortran program, the output from which is formatted in the form of key word card readable by the LS-DYNA3D. In this way, the effects of structural damping and stiffness for the two-layer beam is included in the calculation of the transient response of the beam subjected to underwater shock.

The shock pressure generated by the explosion is determined by the empirical equation [11]

$$
P(t)=P_{\max } e^{-\left(\frac{t-t_{1}}{\theta}\right)} \quad t \geqslant t_{1}
$$

where $t_{1}$ is the instant when the shock arrives, $P_{\max }$ is the peak magnitude of the pressure in the shock front 
Effective modulus ratio, $E_{\mathrm{st}} / E_{1}$

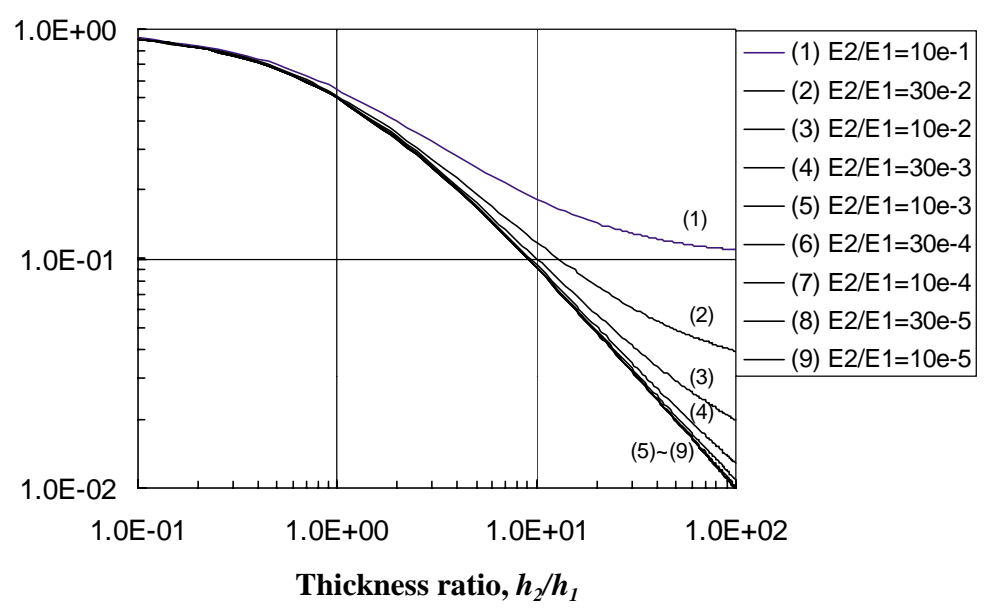

Fig. 3. Variation of relative effective modulus with thickness ratio.

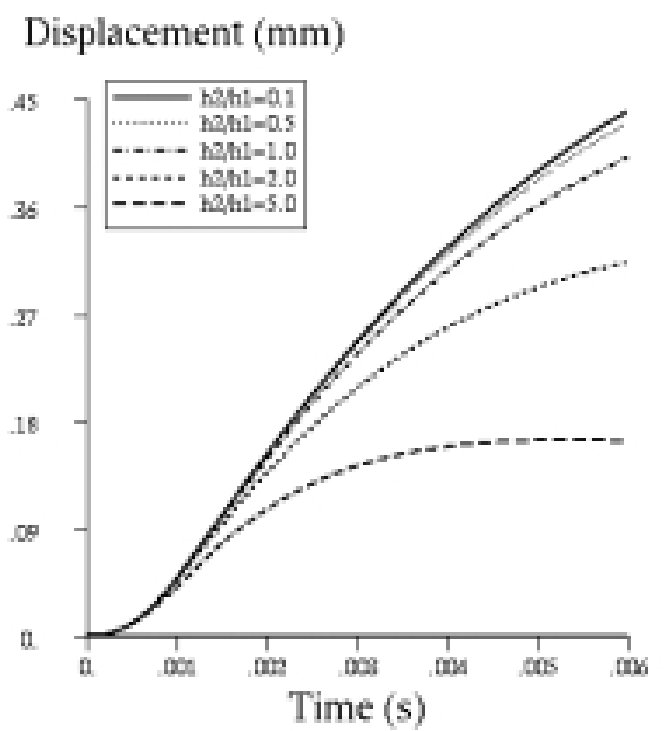

Fig. 4. Variation of central displacement with time for different thickness ratios.

and $\theta$ is the exponential decay constant. The peak pressure and decay constant in Eq. (18) are given by

$$
\begin{aligned}
& P_{\max }=K_{1}\left(W_{c}^{1 / 3} / R\right)^{A_{1}} \\
& \theta=K_{2} W_{c}^{1 / 3}\left(W_{c}^{1 / 3} / R\right)^{A_{2}}
\end{aligned}
$$

where $K_{1}, K_{2}, A_{1}$ and $A_{2}$ are constants which depend on explosive charge type; $W_{c}$ is the weight of the explosive charge and $R$ the distance from the explosive

\section{Velocity $(\mathrm{m} / \mathrm{s})$}

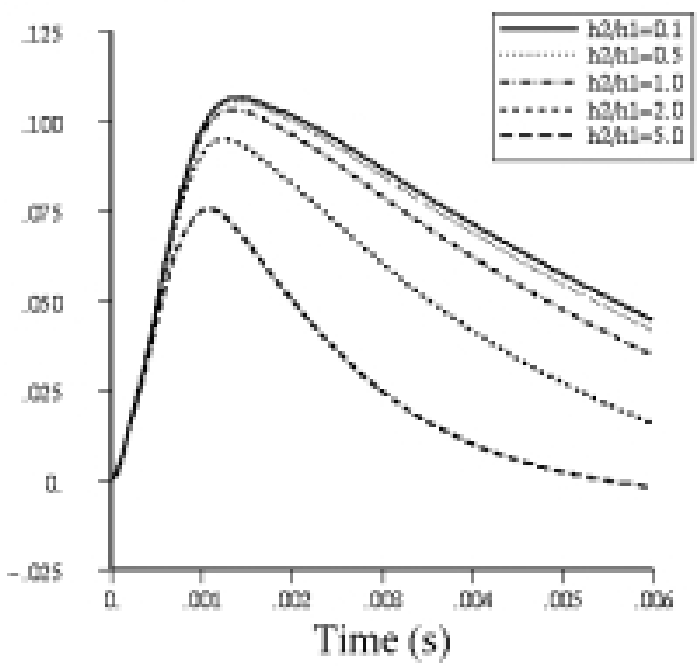

Fig. 5. Variation of central velocity with time for different thickness ratios.

charge to the target. The coupled finite element and DAA boundary element codes perform the staggered time integration to solve the fluid-structure-coupled equations.

\section{Results and discussion}

The layered beam has a length of $1 \mathrm{~m}$, a width of $0.1 \mathrm{~m}$ and a total thickness of $0.04 \mathrm{~m}$. The material 


\section{Strain energy density $\left(\mathrm{N} . \mathrm{m} / \mathrm{m}^{+\infty} 3\right)$}

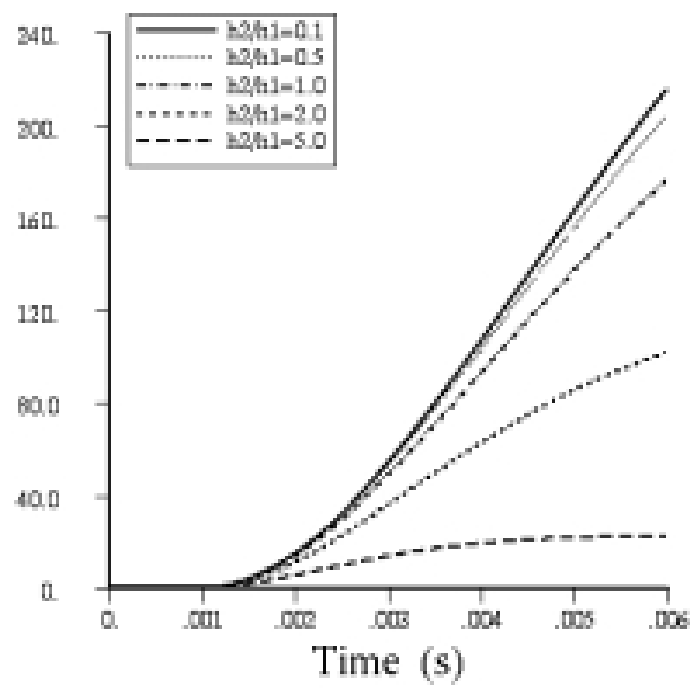

Fig. 6. Variation of internal energy density with time for different thickness ratios.

properties of the basic layer are: $E_{1}=210 \mathrm{GPa}, \nu_{1}=$ 0.3 and $\rho_{1}=7850 \mathrm{~kg} / \mathrm{m}^{3}$. The Poisson's ratio and the density of the damping layer are assumed to be 0.4 and $980 \mathrm{~kg} / \mathrm{m}^{3}$ while the Young's modulus of it varies with the modulus ratio $\lambda_{e}=E_{2} / E_{1}$. In this study, the thickness of the base layer is kept constant while the thickness of the damping layer varies with the thickness ratio $\lambda_{h}=h_{2} / h_{1}$. The effective Young's modulus and Poisson ratio for the layered beam are calculated using Eqs (13) and (14).

Firstly, the influence of the ratio of the damping layer thickness to the base layer thickness on the structural damping and stiffness is investigated. Figure 2 illustrates the variations of the loss factor ratio $\eta_{s t} / \eta_{2}$ with the thickness ratio $\lambda_{h}=h_{2} / h_{1}$ for several modulus ratios $E_{2} / E_{1}$. It is observed that for small thickness ratio $\lambda_{h}$, the relative loss factor $\eta_{s t} / \eta_{2}$ is proportional to the damping layer thickness, whereas for very large thickness ratio the effective loss factor approaches unity; i.e., the loss factor of the two-layer composite beam approaches that of the damping layer. As also is evident from the Fig. 2, at the small thickness ratio, the effective loss factor ratio increases with the modulus ratio $\lambda_{e}=E_{2} / E_{1}$.

Inspection of Fig. 2 shows that for a smaller modulus ratio, increasing the thickness of damping layer has no any effects on the shock attenuation of the layered beam. For example, when $\lambda_{e}=E_{2} / E_{1} \leqslant 1.0 e-$ 5 , even though the thickness of the damping layer is

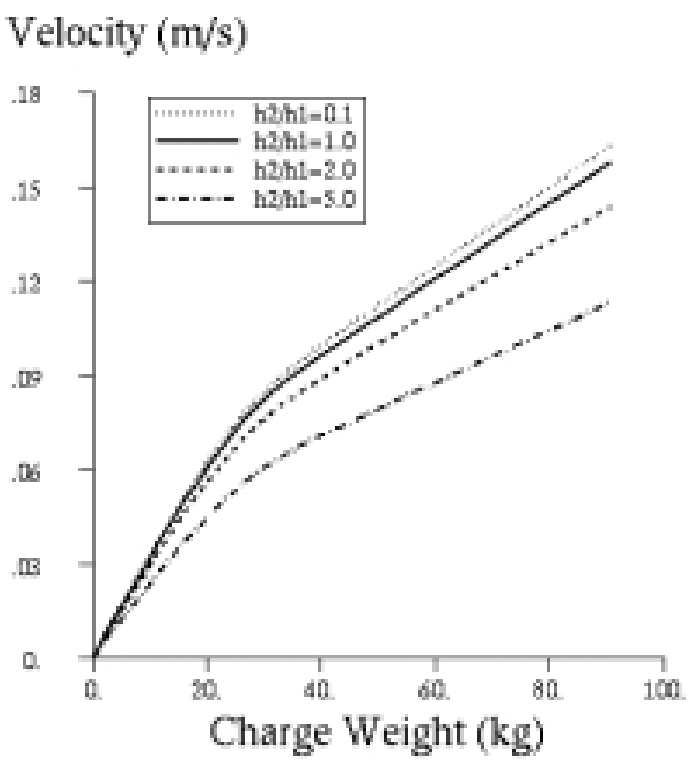

Fig. 7. Variation of central velocity with charge weight for different thickness ratios.

increased to 3 times of the thickness of the basic elastic layer, i.e. $\lambda_{h}=3$, the effective loss factor for the layered beam $\eta_{s t}$ is kept only one thousandth as much as the loss factor of the damping layer $\eta_{2}$. It follows that only for larger modulus ratio $\lambda_{e}$, the loss factor ratio increases with the thickness ratio, in the other word, only for a larger modulus ratio, the damping effects can be enhanced by increasing the thickness of the damping layer. This result implies the optimal damping material should have both high modulus and high loss factor.

The variation of the relative effective modulus for the two layer composite beam with the material and geometry parameters $\lambda_{h}$ and $\lambda_{e}$ is plotted in Fig. 3. In this study, the modulus ratio $\lambda_{e}$ ranges from $10 e-5$ to $10 e-1$. For smaller thickness ratios, $\left(\lambda_{h} \leqslant 1.0\right)$, the relative effective modulus decreases slowly as the thickness ratio increases. However for large thickness ratios, $\left(\lambda_{h} \geqslant 10\right)$, the relative effective modulus decreases much more rapidly. At the thickness ratio $\lambda_{h}=10$, the relative effective modulus for $\lambda_{e}=10 e-1$ is about twice as much as that for $\lambda_{e}=10 e-5$. Further more, at the thickness ratio $\lambda_{h}=100$, the relative effective modulus for $\lambda_{e}=10 e-1$ is about 11 times as much as that for $\lambda_{e}=10 e-5$. This result suggests that the small thickness ratios should be used in the structural design of the two layer composite beam to avoid reducing greatly the effective stiffness of the layered beam.

Transient responses of the two layered beam subjected to underwater shock were investigated. The 


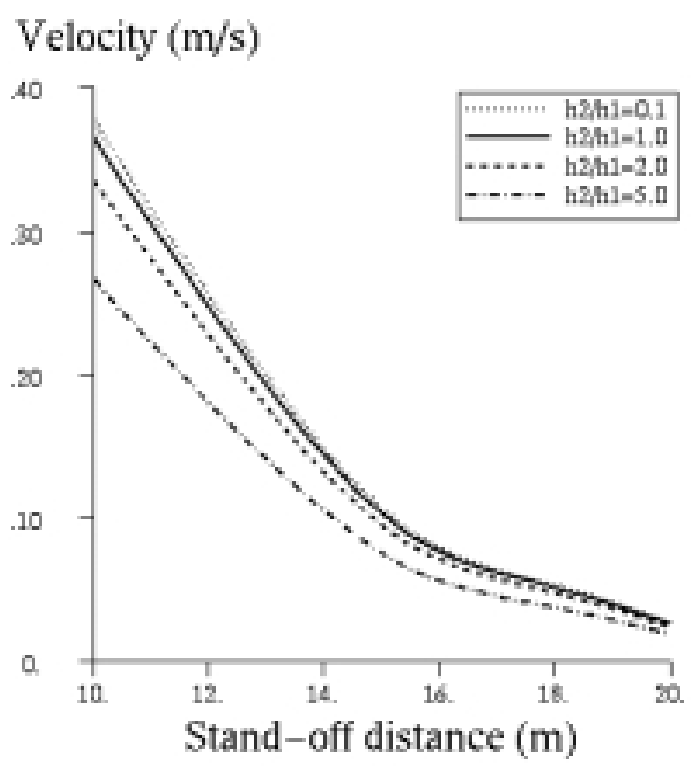

Fig. 8. Variation of central velocity with stand-off distance for different thickness ratios.

charge locates below the center of the beam with a standoff distance of $15 \mathrm{~m}$ and a weight of $50 \mathrm{~kg}$. In this study, the modulus and the thickness ratios are assumed to be $\lambda_{e}=E_{2} / E_{1}=1.0 e-3$ and $\lambda_{h}=H_{2} / h_{1}=1.0$ respectively; and the loss factor of the damping layer $\left(\eta_{2}\right)$ is 0.2 . Figures 4 and 5 show the variation of the displacement and velocity at the center of the layered beam with time for different thickness ratio It is seen that the displacement and velocity at the beam center decrease as the thickness ratio increases. It is obvious that a larger thickness ratio corresponds to a larger effective loss factor as shown in Fig. 2 and thus yields smaller central displacement and velocity. Figure 6 shows the variation of strain energy density with time for different thickness ratio $\lambda_{h}=h_{2} / h_{1}$. The strain energy density is reduced greatly as the thickness ratio increases. The value of the strain density for the thickness ratio $\lambda_{h}=0.1$ at time $t=6 \mathrm{~ms}$ is about 10 times as much as that for the thickness ratio $\lambda_{h}=5.0$. This indicates that the strain energy can be significantly dissipated for larger thickness ratios.

The effects of the charge weight and its distance from the beam center (standoff distance) on the velocity in $z$-direction (Fig. 1) at the beam center were examined. Figures 7 and 8 show the variation of the velocity at the beam center with the charge weight and stand-off distance. The velocities increase exponentially with the charge weight and decrease hyperbolically with the stand-off distance for all cases of the thickness ratios.
Free Surface

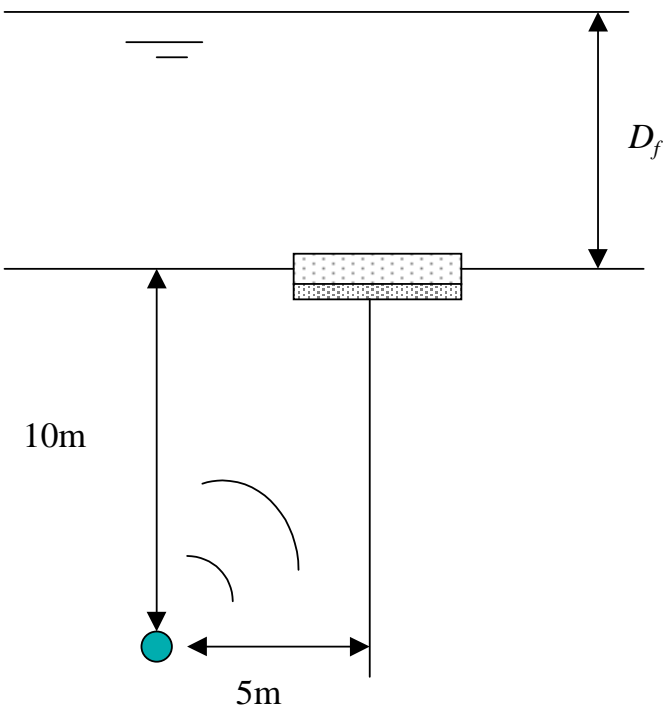

Fig. 9. Schematic diagram of the ship section subjected to underwater explosive shock.

This illustrates that the variation of the velocity with the charge weight and standoff distance is greatly dominated by the peak incident shock pressure described in Eq. (18).

Figure 9 illustrates that a layered beam with a distance $D_{f}$ from the free surface. The layered beam also has a length of $1 \mathrm{~m}$, a width of $0.1 \mathrm{~m}$ and a total thickness of $0.04 \mathrm{~m}$. It is subjected to underwater shock, generated by a charge of of TNT which is detonated underwater with a distance of $11 \mathrm{~m}$ from the beam center. The effects of distance from the free surface to the center of the beam $D_{f}$ on the transient response of the two-layered beam to underwater shock is examined. In this study, the charge weight and the stand-off distance are kept constant.

Figure 10(a) shows the profile of the pressure-time history based on the empirical Eq. (18) without including the effects of free surface. Figure 10(b) shows the profile of pressure-time history in the presence of the free surface, in which $t_{I}$ represents the instant when the image wave arrives. The duration $t_{1}$ may change as either charge location or the measuring point. It depends on the difference between the charge location to the measuring point and the image origin to the same measuring point. The duration $t_{1}$ increases as the difference increases. The reflection of the pressure wave at a free surface arises as the shock wave arrives at the free surface. In the acoustic approximation, the pressure at any point in water is the sum of the pressures in 


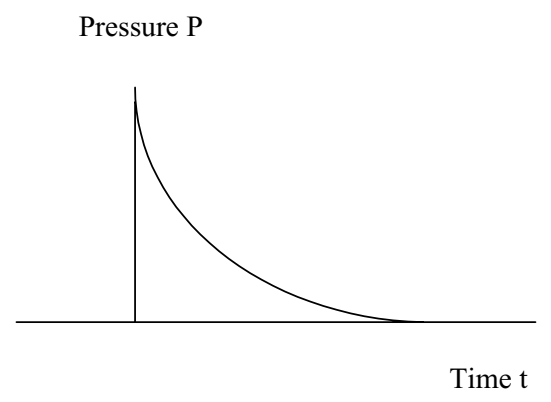

(a) Without free surface

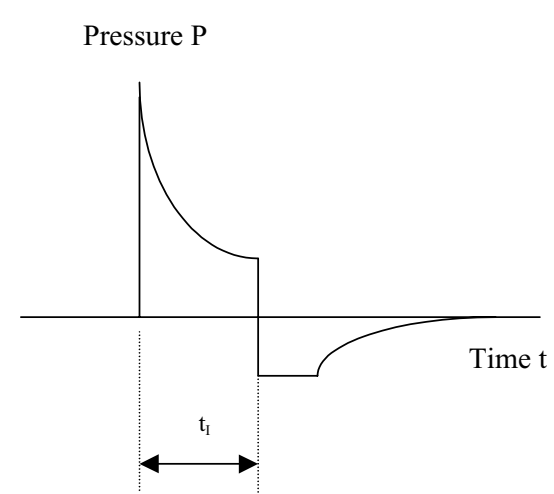

(b) With free surface

Fig. 10. Time history of shock pressure with and without free surface.

\section{Stress (MPa)}

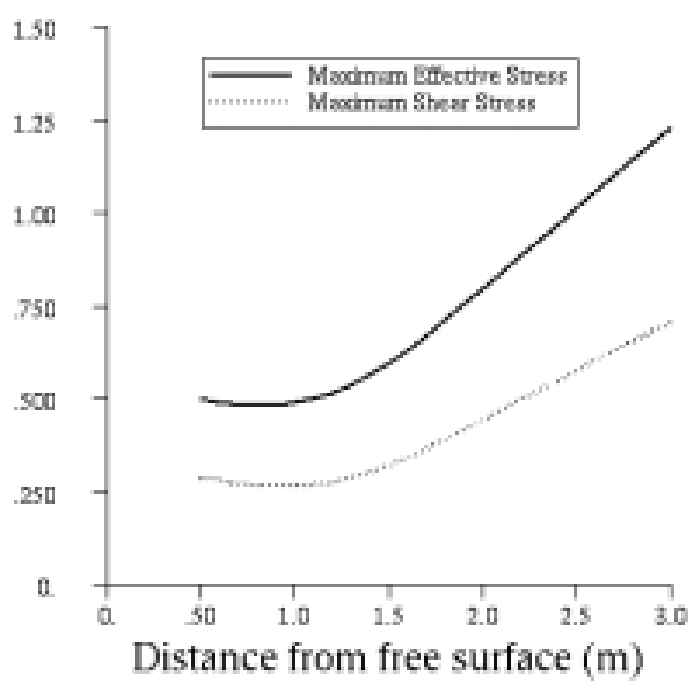

Fig. 11. Variation of maximum stresses with the distance from free surface to the layered beam.

the wave from the charge and the wave from its mirror in air.

Figure 11 shows the effects of the distance from free surface to the layered beam on the stress response at the beam center. In this study, the modulus and the thickness ratios are also assumed to be $\lambda_{e}=E_{2} / E_{1}=$ $1.0 e-3$ and $\lambda_{h}=h_{2} / h_{1}=1.0$ respectively; and the loss factor of the damping layer $\left(\eta_{2}\right)$ is 0.2 . It is seen that the value of the stress increases with the distance from the free surface to the layered beam due to the fact that the duration of $t_{I}$ increases with the distance $D_{f}$ (See Fig. 11). This results indicates that the free surface has a significant effect on shock attenuation when the layered beam close to it.

\section{Conclusions}

A numerical approach was proposed for prediction of transient response of layered beam subjected to underwater shock. In the present approach, the Doubly Asymptotic Approximation (DAA) is employed, in which the effects of effective structural damping and stiffness are incorporated. The present approach facilitates investigation of effects of structural damping and stiffness on transient response of layered structures subjected to underwater shock. The results show that the structural damping has a significant effect on the underwater shock response. The results also illustrate that the optimal damping material should have both high modulus and high loss factor. In addition, the examination of effects of free surface shows that the transient shock can be attenuated significantly when the layered beam is close to it.

\section{References}

[1] T.L. Geers, Residual Potential and Approximate Methods for Three-dimensional Fluid-Structure Interaction Problems, Journal of Acoustical Society of America 49 (1971), 15051510 .

[2] R.W. McCoy and C.T. Sun, Fluid-structure interaction analysis of a thick-section composite cylinder subjected to underwater blast loading, Composite Structures 37 (1997), 45-55.

[3] S.W. Gong and K.Y. Lam, Transient response of stiffened composite submersible hull subjected to underwater explosive shock, Composite Structures 41 (1998), 27-37. 
[4] K.Y. Lam, Z.J.Zhang, S.E. Gong and E.S. Chen, Transient Response of a Two-Layered Elastic Cylindrical Shell Impinged by an Underwater Shock Wave, Composites Part B: engineering 29B (1998), 673-685.

[5] K.Y. Lam, Z.J. Zhang, S.W. Gong and E.S. Chen, Transient Response of Orthortropic Composite Cylinder Exposed to Underwater Shock Wave, Composite Structures 43 (1998), 179 194.

[6] S.W. Gong and K.Y. Lam, Transient Response of Composite Ship Section Subjected to Underwater Explosive Shock, Composite Structures 46 (1999), 65-71.

[7] H.J. Oberst, Ueber die Dampfung der Biegeschwingungen dunner Bleche durch fest haftende Blage, Akustische Beihefte, Acustica 2(4) (1952).

[8] J. DeRuntz, The underwater shock analysis code and its appli- cations, in: Proc. of the 60th Shock and Vibration Symposium 89, Maryland, USA, 1989.

[9] J. Hallquist, LS-DYNA3D Theoretical Manual, Livermore Software Technology Corporation, USA, 1994.

[10] LS-DYNA3D User's Manual, Version 936, Livermore Software Technology Corporation (LSTC), Livermore, CA 94550, 1995.

[11] Y.S. Shin and T.L. Geers, Response of Marine Structures to Underwater Explosions, International Short Course Notebook, Shock and Vibration Research, Monterey, CA, 1994, 3.2-4.

[12] Z. Zong, K.Y. Lam and G.R. Liu, Numerical Investigations of Damaging Effects of Underwater Shock on Submarine Structures in the Presence of Free Surface, 69th Shock and Vibration Symposium, St. Paul, MN, US, 12-16 Oct 1998. 

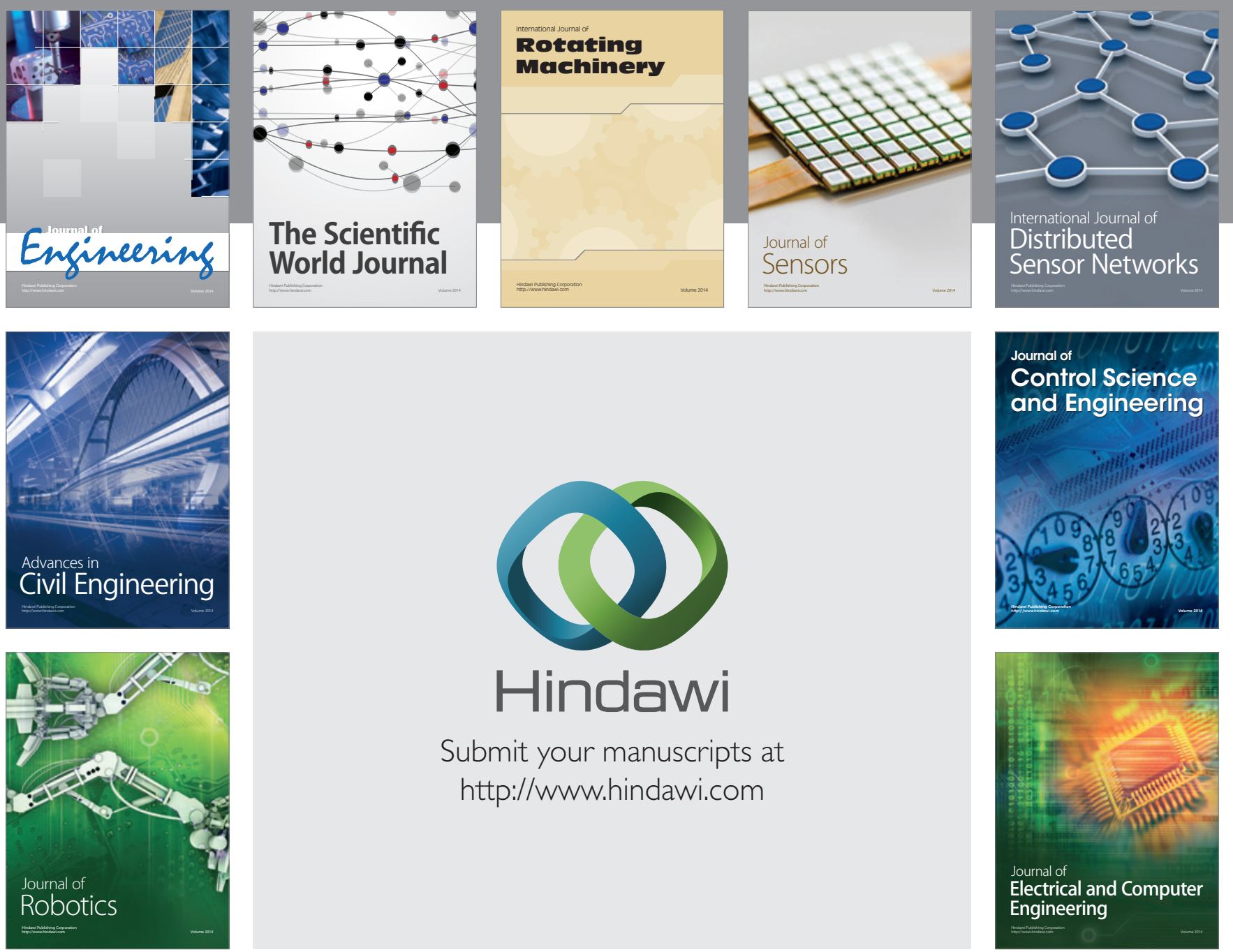

Submit your manuscripts at

http://www.hindawi.com
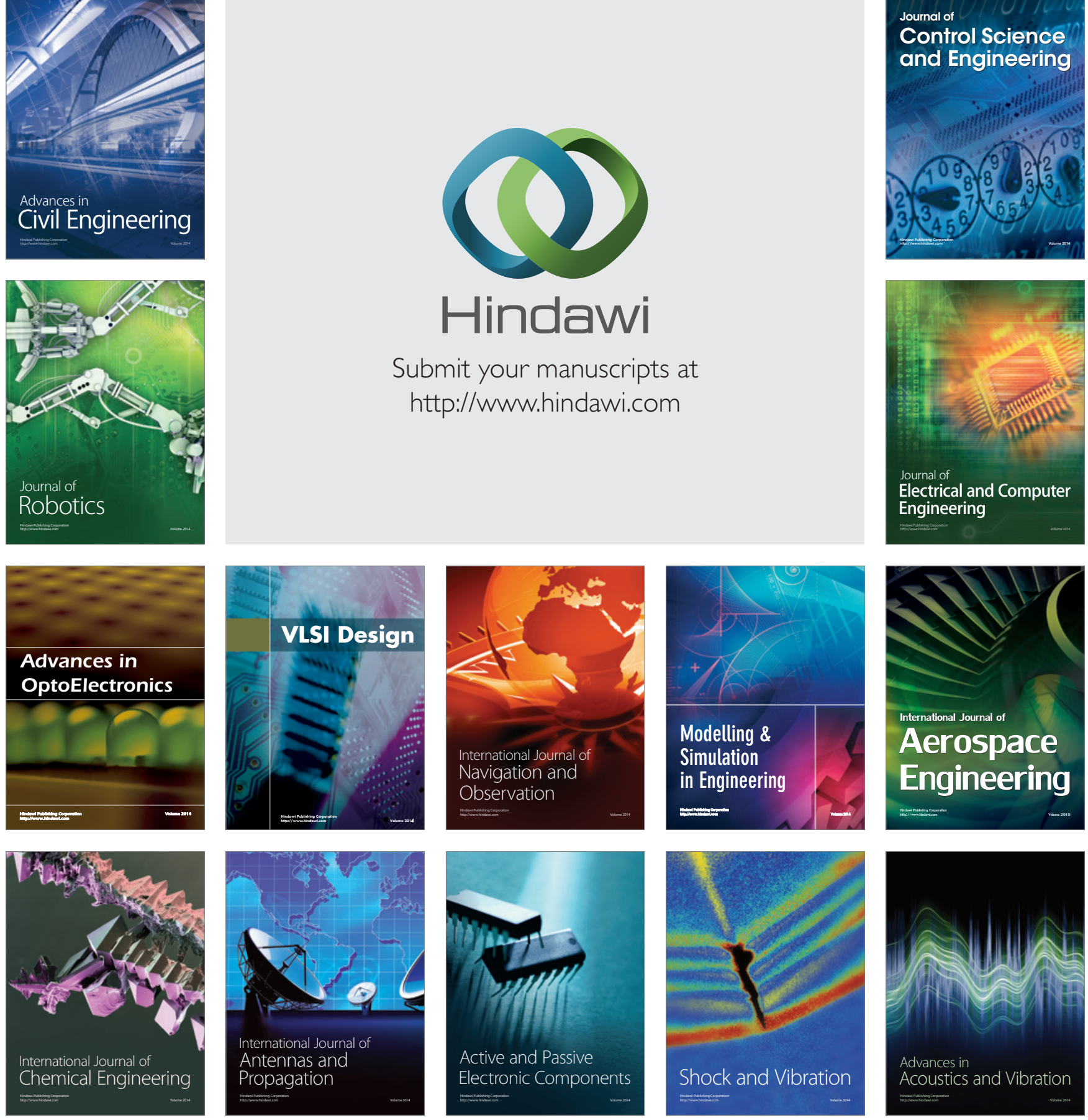